\title{
We need smarter trigger tools for diagnosing sepsis in children in Canada
}

\author{
J. Mark Ansermino MBBCh MMed, Matthew O. Wiens PharmD PhD, Niranjan Kissoon MBBS
}

Cite as: CMAJ 2018 September 10;190:E1060-1. doi: 10.1503/cmaj.180434

CMAJ Podcasts: author interview at https://soundcloud.com/cmajpodcasts/180434-com

$\mathbf{T}$

he failure to recognize and treat the child with sepsis is devastating to the child and family, as well as for physicians, other health care providers and policy-makers. Several recent high-profile deaths from sepsis in Canadian children ${ }^{1}$ have highlighted the need for rapid and reliable identification of early manifestations of sepsis and timely optimized delivery of supportive interventions. A resolution from the World Health Assembly in 2017 highlighted the need to prioritize recognition and early treatment of sepsis, following increasing recognition that sepsis is an important cause of morbidity and mortality globally. ${ }^{2}$ However, this is a challenge in Canada, where the incidence of pediatric sepsis is low, and screening to identify cases is burdensome. We need novel approaches to identify sepsis in children, which can be applied broadly and be well integrated into routine care.

Because most Canadian physicians do not encounter children with sepsis frequently and are not confident in anticipating or diagnosing sepsis, automatic trigger tools may be the best way to identify children at risk and to support health care teams. Trigger tools for sepsis coupled with treatment protocols have been shown to reduce mortality in specialist pediatric settings. ${ }^{3}$ Some are being implemented across Canada, albeit not uniformly.

At present, trigger tools for sepsis are not well integrated into existing early warning systems and triage tools in Canada, and reluctance to do this is understandable. To ensure a high sensitivity, many of these trigger tools have a low specificity. ${ }^{4} \mathrm{~A}$ high rate of false-positive cases may confer a substantial burden on clinical staff because of unnecessary escalation of care, in particular if time and effort is required for additional documentation.

Current trigger tools for diagnosing sepsis in children, which rely on a combination of vital signs and additional signs or symptoms of organ dysfunction, are subject to several important limitations. There is currently no agreement on the clinical characteristics or vital sign thresholds that should trigger a sepsis screen. ${ }^{5}$ Furthermore, the signs and symptoms associated with early sepsis in children may be subtle and protean, and an important challenge is the interdependency of some variables (e.g., an increase in temperature causing an increase in heart and respiratory

\section{KEY POINTS}

- The reliable identification of children with suspected sepsis is critical to optimize the delivery of supportive interventions.

- Thresholds based on individual vital signs or the summation of vital signs do not identify children with sepsis reliably.

- The use of artificial intelligence tools for the automated identification of sepsis may improve upon paper-based screening tools.

rates). Most current trigger tools use single-variable thresholds or a simple summation of these thresholds. However, a retrospective study involving children in intensive care showed that combined heart rate and blood pressure (the so-called shock index) was equally poor as either heart rate or blood pressure in predicting those at risk of dying of sepsis. ${ }^{6}$

Paper-based screening tools ${ }^{7}$ require time-consuming additional entry of data, use scores that have been reduced to a small number of categorical variables and, in our opinion, are of limited value because of the crude summation of individual categorical scores. These tools usually fail to account for the interaction between variables. A data-driven approach for development and validation of definitions and trigger tools for diagnosing sepsis in children, similar to the approach of the Third International Consensus Definitions for Sepsis and Septic Shock task force, ${ }^{8}$ would maintain the fidelity of variables, account for their interaction and constitute a more useful approach to management of sepsis in children.

When designing data-driven trigger tools, it is important to consider context (e.g., community, emergency department or inpatient setting) and locally available resources (e.g., equipment, tests and expertise). The optimal sepsis trigger tool needs to be rapid, objective, accurate and low cost; must easily integrate into the current workflow of a busy clinical setting; should require minimal training and require minimal additional effort; and offer a clear clinical benefit, particularly in community settings where the prevalence and clinical experience with sepsis is likely to be low. 
Integrating sepsis trigger tools into electronic health records to automate the process of screening seems sensible. However, in Canada and elsewhere, most integrated tools have used the same low-specificity algorithms used for paper-based screening. A recent study from the United Kingdom reported that alert fatigue is a growing problem in primary care because of the overtriggering of integrated sepsis tools. ${ }^{9}$ There is little evidence that alerts for sepsis from primary care electronic records are effective in reducing mortality or length of stay in hospital. Alerts must be designed to be useful for the busy clinician and should be integrated into the electronic health record in such a way as to optimize, rather than impede, clinical care.

There is hope. The electronic computing capability currently found in devices for vital sign measurements and electronic health records can improve the specificity of trigger tools without compromising sensitivity. The same smart technologies used for programming washing machines, automated processing of medical images ${ }^{10}$ and identification of skin cancer ${ }^{11}$ could be applied to optimize screening for sepsis, through the automated combination of clinical symptoms, many clinical signs and key demographic data (e.g., age). The recognition and anticipation of sepsis represents an important opportunity for artificial intelligence to revolutionize health care, by optimizing algorithms to a degree of accuracy that would avoid alert fatigue and optimize efficiencies in work flow. This will also require improvement in how outcome data are currently collected and better integration into existing electronic medical records.

We propose that research funders in Canada support a concerted research effort to optimize data collection on sepsis outcomes and develop high-performing screening for sepsis and trigger tools to ensure that Canadian children with or at risk of sepsis are identified and treated quickly without unnecessary burden on the health care provider or referral systems. This same datadriven approach could simultaneously be used to optimize triage, referrals and even follow-up after discharge from hospital. ${ }^{12}$

\section{References}

1. Sepsis can be difficult to recognize, but early diagnosis and treatment is essential. Ottawa: Canadian Medical Protective Association; 2015. Available: www.cmpa-acpm.ca/en/advice-publications/browse-articles/2015/sepsis-can-be -difficult-to-recognize-but-early-diagnosis-and-treatment-is-essential (accessed 2018 Aug. 14)

2. Seventieth World Health Assembly update [news release]. Geneva: World Health Organization; 2017. Available: www.who.int/mediacentre/news/ releases/2017/wha-70/en/ (accessed 2018 Mar. 14).

3. Evans IVR, Phillips GS, Alpern ER, et al. Association between the New York sepsis care mandate and in-hospital mortality for pediatric sepsis. JAMA 2018; 320:358-67.

4. Balamuth F, Alpern ER, Grundmeier RW, et al. Comparison of two sepsis recognition methods in a pediatric emergency department. Acad Emerg Med 2015; 22:1298-306.

5. Scott HF, Deakyne SJ, Woods JM, et al. The prevalence and diagnostic utility of systemic inflammatory response syndrome vital signs in a pediatric emergency department. Acad Emerg Med 2015;22:381-9.

6. Ray S, Cvetkovic M, Brierley J, et al. Shock index values and trends in pediatric sepsis: predictors or therapeutic targets? A retrospective observational study. Shock 2016;46:279-86.

7. BC Sepsis Network. Inpatient sepsis toolkit: speed is life. Vancouver: BC Patient Safety \& Quality Council; 2011. Available: https://bcpsqc.ca/wp-content/ uploads/2018/02/SepsisToolkit_June22b.pdf (accessed 2018 Aug. 14).

8. Singer M, Deutschman CS, Seymour CW, et al. The third international consensus definitions for sepsis and septic shock (Sepsis-3). JAMA 2016;315:801-10.

9. The Lancet Respiratory Medicine. Crying wolf: the growing fatigue around sepsis alerts. Lancet Respir Med 2018;6:161.

10. Hosny A, Parmar C, Quackenbush J, et al. Artificial intelligence in radiology. Nat Rev Cancer 2018;18:500-10.

11. Esteva A, Kuprel B, Novoa RA, et al. Dermatologist-level classification of skin cancer with deep neural networks. Nature 2017;542:115-8.

12. Wiens MO, Kissoon N, Kabakyenga J. Smart hospital discharges to address a neglected epidemic in sepsis in low- and middle-income countries. JAMA Pediatr 2018;172:213-4.
Competing interests: J. Mark Ansermino is the founder of LGT Medical, which developed a prediction tool for preeclampsia, and has an equity stake in the company (less than 5\%). Niranjan Kissoon is Vice-Chair of the Global Alliance for Sepsis. No other competing interests were declared.

This article has been peer reviewed.
Affiliations: Departments of Anesthesiology, Pharmacology and Therapeutics (Ansermino, Wiens), and Pediatrics (Kissoon), Faculty of Medicine, The University of British Columbia; Centre for International Child Health, BC Children's Hospital, Vancouver, BC

Contributors: All of the authors contributed equally to the conception, drafting and critical revision for important intellectual con- tent of the manuscript, gave final approval of the version to be published and agreed to be accountable for all aspects of the work.

Disclaimer: Niranjan Kissoon is a member of the CMAJ Editorial Board and was not involved in the decision-making process for this article.

Correspondence to: J. Mark Ansermino, mansermino@bcchr.ca 